This is the postprint version of the following article: Gallardo A, Martínez-Campos E, García C, Cortajarena AL, Rodríguez-Hernández J. Hydrogels with Modulated lonic Load for Mammalian Cell Harvesting with Reduced Bacterial Adhesion. Biomacromolecules. 2017;18(5):1521-1531. doi: $10.1021 /$ acs.biomac.7b00073. This article may be used for non-commercial purposes in accordance with ACS Terms and Conditions for Self-Archiving. 


\title{
Hydrogels with Modulated Ionic Load for Mammalian Cell Harvesting with Reduced Bacterial Adhesion
}

\author{
Alberto Gallardo, ${ }^{1 *}$ Enrique Martínez-Campos ${ }^{1,2}$, Carolina García, ${ }^{1}$ Aitziber L. Cortajarena ${ }^{3}$ and \\ Juan Rodríguez-Hernández ${ }^{1 *}$
}

1. Instituto de Ciencia y Tecnología de Polímeros (ICTP), Consejo Superior de Investigaciones Científicas (CSIC), C/Juan de la Cierva 3, 28006 Madrid, Spain. Email: Alberto Gallardo: gallardo@ictp.csic.es, Juan Rodríguez-Hernández: jrodriguez@ictp.csic.es

2. Tissue Engineering Group; Instituto de Estudios Biofuncionales, Universidad Complutense de Madrid. Paseo Juan XXIII, nº 128040 Madrid, Spain.

3. (a) IMDEA-Nanociencia and Centro Nacional de Biotecnología (CNB-CSIC) - IMDEA Nanociencia Associated Unit, Universidad Autónoma de Madrid, Cantoblanco, 28049 Madrid, Spain. (b) CIC biomaGUNE, Parque Tecnológico de San Sebastián, Paseo Miramón 182, 20014 Donostia-San Sebastián, Spain (c) Ikerbasque, Basque Foundation for Science, Mª Díaz de Haro 3, 48013 Bilbao, Spain

\section{Abstract}


In this manuscript, we describe the fabrication of hydrogel supports for mammalian cell handling that can simultaneously prevent materials from microbial contamination and therefore allow storage in aqueous media. For that purpose, hydrogels based on the antifouling polymer polyvinylpyrrolidone (PVP) were functionalized with different ionic groups (anionic, cationic or two types of zwitterions). In order to prevent bacterial adhesion in a long term, we took advantage of the synergistic effect of inherently antifouling PVP and additional antifouling moieties incorporated within the hydrogel structure. We evaluated, a separate series of experiments both, the capability of the materials to act as supports for the growth of mammalian cell monolayers for transplantation (using C-166-GFP endothelial cell line), as well their antifouling properties against Staphylococcus aureus, were studied. All of the hydrogels are structurally pseudo-double networks with high swelling (around $90 \%$ ) and similar mechanical properties (in the low range for hydrogel materials with Young modulus below $1250 \mathrm{kPa}$ ). With some differences, all the charged hydrogels were capable of hosting mouse endothelial cell line C166-GFP to confluence, as well as a monolayer detachment and/or transplantation through simple mechanical agitation. On the contrary, the uncharged hydrogel was not capable to detach a full monolayer for transplatation. Bacterial adhesion and proliferation was highly sensitive to the functionality (type of charge and density). In particular, we evidenced that monomers bearing zwitterionic sulfobetaine groups, those negatively charged as well as "electro neutral" hydrogels fabricated from stoichiometric amounts of positive and negative units exhibit excellent antifouling properties both at initial adhesion times and during longer periods up to $72 \mathrm{~h}$.

\section{Keywords}

Hydrogels, antifouling, cell selective, cell manipulation, cell detachment, cell monolayer, double networks, swelling, surface charge

\section{Introduction}


Hydrogels have attracted extensive attention in multiple applications, for instance for drug delivery, as scaffolds in tissue engineering, and have been employed to mimic natural tissues. ${ }^{1}$ Other related applications include their use in regenerative medicine ${ }^{2}$ mainly as barriers, cell encapsulation ${ }^{3}$ or as supports for cell manipulation. ${ }^{4,5}$ As an example of the later, our group has recently reported on the preparation of poly-vinylpyrrolidone (PVP) based hydrogels with pseudo-double network structure and unique properties for cell manipulation, which are based on the incorporation to the PVP structure of an anionic methacrylic unit. ${ }^{4}$ The supports allowed mouse $\mathrm{C} 2 \mathrm{C} 12$ pre myoblastic cells to grow to confluence, and subsequently rapid cell detachment could be induced through simple mechanical agitation and the cell sheets could be transplanted easily without the need for a cell superstrate. The materials were prepared via a simple one step radical photopolymerization, and they were, in the hydrated state, robust and easy to 'handle' and maneuver in despite of the high water uptake (above $85 \mathrm{wt} \%$ ). The term pseudo-double networks $(\mathrm{DN})^{6}$ refers to the structural tendency of these materials to form DNs, which can be described as interpenetrating polymer networks (IPNs) comprised of two highly asymmetric crosslinked networks. The first DN was reported by Gong et al. ${ }^{7}$ and consisted of a first highly crosslinked network of ionic poly(2-acrylamide-2-methyl-propane sulfonic acid) and a second slightly crosslinked network of non-ionic and water soluble polyacrylamide. Many more DNs have been reported since. These DNs must be prepared in a two-step procedure to obtain the first and the second network, respectively. DNs has been reported to exhibit astonishing mechanical properties in the high swollen state. ${ }^{7}$ The pseudo-DN approach described by us was based on the well-known high differential reactivity of $V P$, (a very low activated monomer in radical polymerization) compared to other commercial monomers such as methacrylates $(M)$, i.e. $V P$ is far less reactive than any $M$ in radical copolymerization. The reaction leads to somehow 'self-ordered' chains in which chains richer in $M$ units are formed first, while chains richer in $V P$ are formed in the last steps of the reaction after exhaustion of $M$. The topology of such a two-component heterogeneous network may be controlled by the nature of the crosslinker used. ${ }^{6,8}$ Therefore, upon proper crosslinking, the two types of chains may become the components of the $1^{\text {st }}$ and $2^{\text {nd }}$ network of the pseudo-DN, which are respectively 
ionic ( $M$-rich chains), and neutral and water soluble ( $V P$-rich chains) as the original described by Gong et al. ${ }^{7}$. In this work on PVP-based pseudo-DN, it was shown that pseudo-DN constructed only with the neutral PVP hydrogel -without the anionic methacrylate-, presented poor mammalian cell adhesion. This result is in agreement with the well-known non-fouling character of such type of uncharged and water-soluble polymers (i.e. PVP, or polyethylene glycol, PEG). Interestingly, the incorporation of the anionic methacrylate $M$ (in a $V P / M$ molar ratio of 6/1) improved both cell adhesion and proliferation. However, protein adsorption and mammalian cell adhesion are also influenced by other additional features, including surface charge/hydrophobicity, mechanical environment or water content, etc. [4]

With these precedents, this work is devoted to the study of analogous PVP-based supports bearing other charged commercial methacrylic units in addition to the negative one: positive, zwitterionics (phosphorylcholine and sulfobetaine), and mixture of positive and negative. As the pseudo-DN structure was designed on a differential reactivity basis -between $M$ and $V P$-, it may be hypothetically extended to any $V P-M$ couple, maintaining the molar ratios. A platform of PVP-based hydrogels with different charges and pseudo-DN structure has been synthesized, with two aims:

A first objective is to clarify the role of the different characteristics of the original pseudo DN (elastic modulus, swelling, and negative charge) on the mammalian cell response, i.e. on the monolayer formation and detachment. Mouse endothelial cell line C166-GFP has been used for this study.

A second objective is to study the interactions of this family of hydrogels with bacteria, because some of the charged structures, such as the positively charged quaternary ammonium salts, or the zwitterionic groups 2-methacryloyloxyethyl phosphorylcholine ${ }^{9}$ or poly(sulfobetaine methacrylate $)^{10}$, are well known as antifouling/antimicrobial moieties. Thus, some of the proposed pseudo-DN will combine two antifouling/antibacterial strategies (PVP based structure and charged moieties). The antifouling behavior provided by the neutral components of the hydrogel will be synergistically improved either with the repelling or the antimicrobial activity of the functional groups incorporated within the hydrogel structure. Current antibacterial 
strategies rely on the combination of complementary approaches. This study has notable interest because hydrogels used in the biomedical field still have an important limitation related to their contamination when stored in aqueous media. Hence, microorganism contamination, in particular with bacteria, is a serious drawback for the employment of polymeric materials in medical applications. ${ }^{11}$

The two proposed objectives, collected together, face an ambitious goal that is currently under investigation by several research groups: ${ }^{12}$ the preparation of selective hydrogels exhibiting mammalian cytocompatibility and antibacterial characteristics. To the best of our knowledge efforts to produce hydrogels with the appropriate mechanical properties and tailored bioresponsive functionality have been carried out in parallel depending on the type of cells involved and their final use: mammalian cells for tissue engineering purposes or microorganisms such as bacteria in order to prevent or remove bacteria from the hydrogel surface.

\section{Experimental Part}

\section{Materials}

[2-(Methacryloyloxy)ethyl]trimethylammonium chloride solution $\left(M-N^{+}\right)$, potassium sulfopropylmethacrylate $\left(\mathrm{M}_{-} \mathrm{SO}_{3}^{-}\right)$, 2-methacryloyloxyethyl phosphorylcholine $\left(\mathrm{M}-\mathrm{PO}_{3}^{-}-\mathrm{N}^{+}\right)$, [2(methacryloyloxy)ethyl]dimethyl-(3-sulfopropyl)ammonium hydroxide $\quad\left(\mathrm{M}^{-} \mathrm{N}^{+}-\mathrm{SO}_{3}{ }^{-}\right)$, vinylpyrrolidone $(V P)$, ethylene glycol dimethacrylate $(C 1)$, 1-hydroxyl cyclohexyl phenyl ketone $(H C P K)$ and azobisisobutyronitrile $(A I B N)$ were purchased from Sigma. $V P$ was distilled prior to use and stored at $4^{\circ} \mathrm{C}$. $A I B N$ was recrystallized from ethanol and stored at $4^{\circ} \mathrm{C}$. The divinylic compound $C 2$ used as second crosslinker, which is a $V P$ derivative, was synthesized in our laboratory as previously described. ${ }^{13}$

\section{Networks synthesis}


A previously optimized formulation ${ }^{4}$ was used as starting point in all cases: water as solvent, $V P$ and $M$ concentrations of 6 and $1 \mathrm{~mol} / \mathrm{L}$ respectively, $V P / C 2$ and $V P / C 1$ molar ratios of always 1000 and 50 respectively. In the case of the networks containing the zwitterionic components, a concentration of $M=0.5 \mathrm{~mol} / \mathrm{L}$ was also prepared. Hydrogels were synthesized in a one-step conventional radical photopolymerization using $H C P K$ as initiator $(0.5 \mathrm{wt} \%)$. The reaction mixtures were bubbled with $\mathrm{N}_{2}$ and transferred to polypropylene molds via syringe. The polypropylene molds were separated by silicone spacers of $0.5 \mathrm{~mm}$ thick. The polymerization was carried out for 40 minutes under UV radiation $(\lambda=365 \mathrm{~nm})$ in a UVP ultraviolet lamp (model CL-1000L, 230V). A summary of the hydrogels types prepared are given in Table 1.

The networks were recovered from the molds and were allowed to swell in Milli-Q water until equilibrium was reached. Subsequently, they were exhaustively washed with water to remove any soluble material and finally they were cut into cylinders. Samples were stored in ethanol at $4^{\circ} \mathrm{C}$ until needed for experimentation. 24 hours before the different experiments, samples were again transferred to Milli-Q water and washed several times to remove completely the ethanol.

\section{Characterization}

\section{Hydrogel swelling}

The swollen cylinders were accurately weighted and then allowed to dry until a constant weight was reached. The swelling $(S)$ was defined as the amount of water per gram of dry network in the equilibrium state (equation 1).

$$
S=\frac{\left(W_{h}-W_{d}\right)}{W_{d}}
$$

Where $W_{h}$ is the swollen weight and $W_{d}$ is the dried weight.

\section{Mechanical properties of the hydrogels}


Mechanical properties were measured by dynamic compression test. Measurements were performed on gels hydrated at equilibrium, at $25^{\circ} \mathrm{C}$, immersed in water, and using a universal test system (MTS System) in compression mode. Cylindrical samples with diameter of $6 \mathrm{~mm}$ and thickness of around $0.8 \mathrm{~mm}$ were used. Samples were placed between compression platens of which the upper one is $3 \mathrm{~mm}$ of diameter. Each sample was subsequently deformed to a specific compressive strain level $(15 \%)$ at $0.1 \mathrm{~mm} / \mathrm{min}$. All measurements were performed by quintuplicate.

\section{Surface properties}

The surface wettability of the hydrogels was measured by the capillary rise technique. ${ }^{14}$ A 0.3 $\mathrm{cm} \times 2 \mathrm{~cm}$ hydrogel piece was place vertical to an aqueous solution. When the hydrogel contacts the surface of the water a capillary quickly rises along the vertical hydrogel. ${ }^{15}$ According to the literature the height of the meniscus is an indirect measurement of the hydrophilicity of a plate, in this case the hydrogel. ${ }^{16}$ The height of the capillary rise $h$ can be measured and by integration of the Laplace equation ${ }^{17}$ result the following equation 2 :

$$
\sin \theta=1-\frac{\Delta \rho g h^{2}}{2 \gamma_{l v}}
$$

where $\Delta \rho$ is the difference in density between the liquid (water in this case $1000 \mathrm{~kg} / \mathrm{m}^{3}$ ) and vapor (air $\left.1.09 \mathrm{~kg} / \mathrm{m}^{3}\right) . g$ is the acceleration due to gravity $\left(9,8 \mathrm{~m} / \mathrm{s}^{2}\right)$ and $\gamma_{l v}$ is the liquid surface tension $\left(72.75 \mathrm{mN} / \mathrm{m}^{2}\right.$ for water). Finally, $h$ is the capillary rise and $\theta$ is the contact angle. As a result, a direct measurement of the capillary rise can be employed to calculate the contact angle. The temperature of the water solution was maintained constant at $25^{\circ} \mathrm{C}$.

\section{Experimental protocol for the cell-adhesion experiments.}

Prior to cell studies, all hydrogels were sterilized with a $70 \%$ ethanol solution rinsing four times during 10 minutes. Then, the hydrogels were washed with PBS four times, exposed to UV 
radiation during 20 minutes, washed two times with incomplete culture medium (DMEM), and finally washed two times with complete culture medium (FBS and antibiotics).

The cell studies were carried out using C166-GFP, a mouse endothelial cell line (CRL 2586 ${ }^{\mathrm{TM}}$, obtained from ATCC ${ }^{\circ}$, USA) derived by transfection with a plasmid reporter vector, pEGFPN1, encoding enhanced green fluorescent protein (GFP). Routine passaging of the cell line was performed with DMEM high in glucose (GIBCO, UK), supplemented with $10 \%$ fetal bovine serum (FBS, 10500-064, Gibco, UK) plus antibiotics (100 U/mL penicillin, $100 \mu \mathrm{g} / \mathrm{mL}$ streptomycin sulfate and $0.2 \mathrm{mg} / \mathrm{ml} \mathrm{G418)} \mathrm{(Gibco,} \mathrm{UK;} \mathrm{Sigma,} \mathrm{USA).} \mathrm{As} \mathrm{a} \mathrm{result} \mathrm{of} \mathrm{their} \mathrm{self-}$ fluorescence, they can be analyzed through some non-opaque/translucent surfaces, such as the hydrogels employed here.

For culturing cells over the hydrogels, cells were seeded singly on the samples with a density of $3 \times 10^{4} /$ hydrogel in supplemented DMEM. Hydrogels were placed in a 24 -well plate in maintenance medium, incubated at $37^{\circ} \mathrm{C}$ with $5 \% \mathrm{CO}_{2}$ in a humidified incubator. For cell detachment experiments, hydrogels were moved to a new 24-well plate with surface treatment for cell culture and supplemented DMEM, turning upside down the hydrogel and putting in contact the cell monolayer with the plastic surface for at least 4 hours. After that, hydrogels were removed.

\section{Metabolic activity study: Alamar Blue assay.}

Metabolic activity of cells was measured by Alamar Blue assay, this was performed following the manufacturer's instructions (Biosource, CA, USA). Assays were performed in triplicate on each sample type. This method is non-toxic, scalable and uses the natural reducing power of living cells, generating a quantitative measure of cell viability and cytotoxicity. Briefly, Alamar Blue dye (10\% of the culture volume) was added to each well, containing living cells seeded over films, and incubated for 90 minutes. The fluorescence $\left(\lambda_{\text {ex }} / \lambda_{\text {em }} 535 / 590 \mathrm{~nm}\right)$ of each well was measured using a plate-reader (Synergy HT, Brotek). 


\section{Bacterial adhesion tests}

S. aureus strain RN4220 carrying the plasmid pCN57 for Green fluorescent protein (GFP) expression (generous gift from Iñigo Lasa's Laboratory at Instituto de Agrobiotecnología, UPNA-CSIC-Gobierno de Navarra) was grown overnight at $37^{\circ} \mathrm{C}$ in Luria-Bertani (LB) media with erythromycin $(10 \mu \mathrm{g} / \mathrm{ml})$. The cells were centrifuged and washed three times in PBS buffer (150 mM NaCl, $50 \mathrm{mM}$ Na-phosphate $\mathrm{pH}$ 7.4). The solution was adjusted to an optical density (OD) at $600 \mathrm{~nm}$ of 1.0 using an UV-VIS Varian Cary 50 spectrophotometer.

The different hydrogels were incubated during 1 hour with the bacterial suspension at $\mathrm{OD}=1.0$, that corresponds to approximately $1-1.510^{9} \mathrm{cfu} / \mathrm{ml}$, in PBS buffer with $0.05 \%$ Tween 20 to allow for bacteria adhesion. After incubation the surfaces were washed with PBS three times during 15 minutes in order to remove the non-adhered bacteria.

Bacteria adhesion to the different surfaces was then monitored and quantified by fluorescence microscopy using a Leica DMI-3000-B fluorescence microscope using the green fluorescence signal of the cells. Images were acquired using x 20 magnification and the corresponding set of filters for imaging green fluorescence and bright field.

The next step was to assess the effect of the surface composition on the bound bacteria, and the potential inhibition on the biofilm formation and bateriostatic activity. For this purpose, the surfaces obtained after $1 \mathrm{~h}$ incubation with bacteria were immersed in $2 \mathrm{ml}$ of $\mathrm{LB}$ media and incubated at $37^{\circ} \mathrm{C}$ during longer time periods up to $72 \mathrm{~h}$ to permit the growth of the immobilized bacteria. After the incubation time the surfaces were carefully washed by immersion into PBS buffer and the amount of bacteria on the surface was imaged by fluorescence microscopy as described above.

\section{Results and discussion}

Fabrication of pseudo-double networks based on VP: wettability and mechanical 


\section{properties}

The hydrogels prepared by incorporation of functional monomers are summarized in Table 1. It is worth to mention that the replacement of $M$ in the formulation does not have a dramatic influence on the swelling and mechanical properties of the hydrogels. Swelling is high and close to $90 \%$ in all cases, while the compression moduli are in the same order of magnitude and belong to the middle-soft range of hydrogel materials. The control hydrogel with no $M$ exhibits the lower modulus, which is in agreement with the results obtained previously. ${ }^{4}$ 
Table 1. Properties and structural characteristics of the PVP based hydrogels with variable charge.

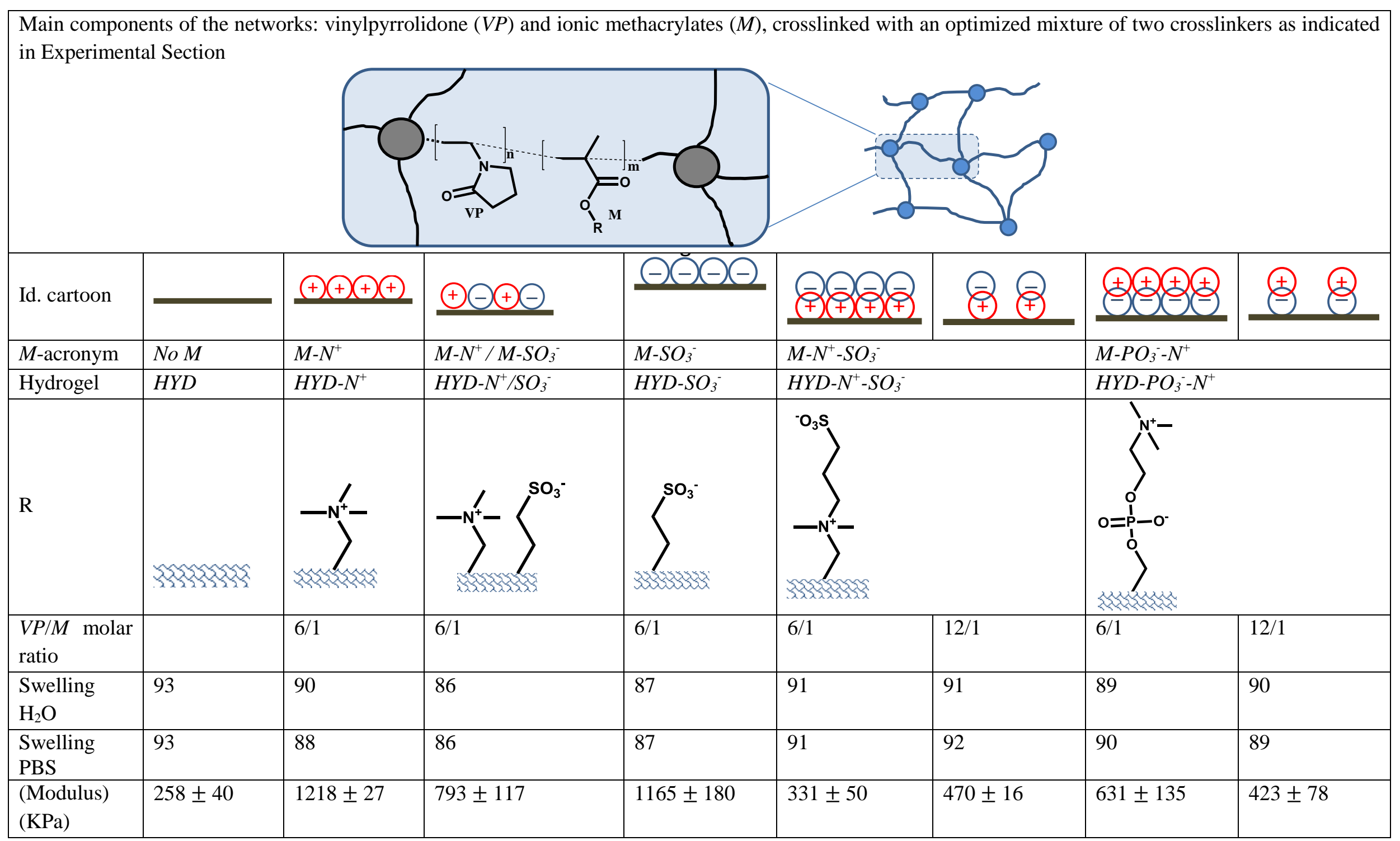


The surface wettability was measured by using the capillary rise approach.${ }^{14}$ For that purpose, as depicted in Figure 1(a), the hydrogels were fixed on a $z$-moving part. When in contact with the solution, the water rises due to the capillary effect. Interestingly, as has been previously reported, this capillary effect is directly related to the dynamic contact angle. ${ }^{15}, 16$ Contact angle measurements using this procedure can be achieved by measuring the capillary rise (or meniscus height) and introducing the value in the integrated form of the Laplace equation ${ }^{17}$ (see experimental section). This methodology is particularly suited for hydrogels since neither static nor advancing and receding contact angles can be measured by the sessile drop approach. The capillary rise values obtained for the different hydrogels are represented in Figure 1(b), as a function of the chemical composition of the different hydrogels explored (schematically represented based on the charge incorporated). The meniscus height obtained for all the hydrogels prepared remain rather high between 1.4 and $1.7 \mathrm{~cm}$. As a result, these values, when introduced in the Laplace equation resulted in contact angles close to 0 independently of the hydrogel analyzed. We can, thus, conclude that all the systems explored showed a highly hydrophilic surface based on the dynamic contact angle measurements, in agreement with the high swelling values depicted in Table 1.

(a)



(b)




Figure 1. (a) Illustrative image of the meniscus height measurement of a hydrogel. (b) Capillary rise (meniscus height) for the hydrogels in water as a function of the surface chemical composition.

Besides hydrophilicity, substrate stiffness plays a key role on the adhesion both of mammalian cells and bacteria as has been depicted in previous reports. ${ }^{18-20}$ Taking this into account, in this study the Young modulus of the different hydrogels was measured by dynamic compression test. As quoted in Table 1 the values of the Young moduli measured were in the same order of magnitude, varying between 260 and $1200 \mathrm{kPa}$. Therefore according to Kolewe et $\mathrm{al}^{21}$ and Guégan et al. ${ }^{22}$ all the hydrogels prepared can be classified as soft hydrogels. As a result, in the systems considered herein, cell adhesion (bacterial and endothelial mouse cells) will be mainly determined by the chemical composition (type of charge and density) rather than by the hydrophilicity or the mechanical properties of the interface.

\section{Adhesion, proliferation and monolayer detachment of mammalian cells}

In order to analyze the role of the surface charge on the ability of mammalian cells to grow on the hydrogels, C166-GFP endothelial cells were seeded over all surfaces. As depicted in Figure 2, after 96 hours growth over these materials, small cell clumps and patches were observed. However, these cell clumps were able to grow and finally develop a cell monolayer after $168 \mathrm{~h}$. In comparison to tissue control plate control (TCP), cell culture growth was delayed in almost all samples (except for positively charged at early stages). These results were also observed in previous results carried out with mouse $\mathrm{C} 2 \mathrm{C} 12$ cells cultured on hydrogels prepared using negatively charged monomers ${ }^{4}$. 


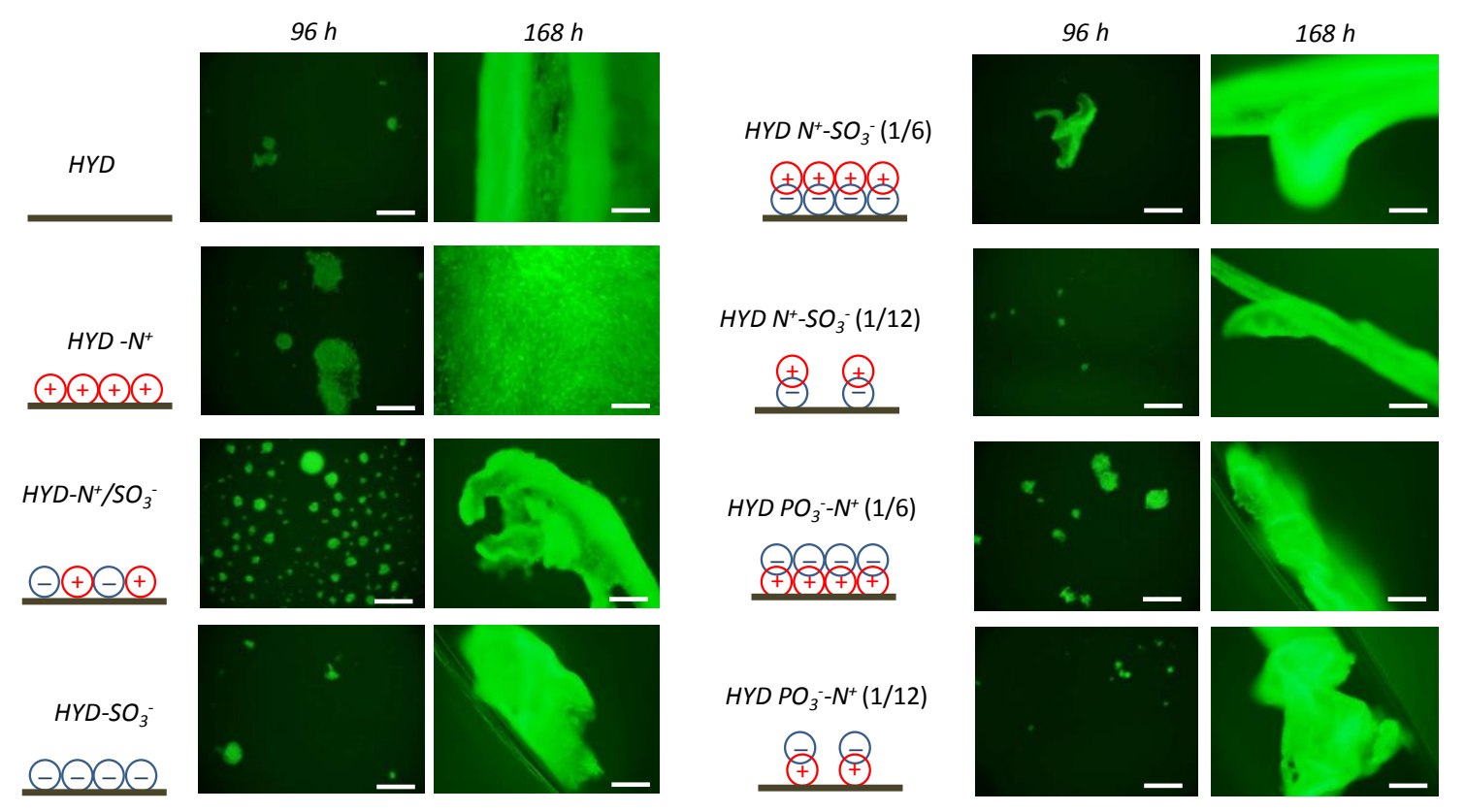

Figure 2. Fluorescence images of cell culture over hydrogels with variable surface charge (96 and 168 hours). At day 4, cell clumps and patches were detected over all kind of surfaces. At day 7, well-grown cell monolayers were observed, presenting a low attachment to the hydrogels. (Scale bar: $200 \mu \mathrm{m})$

In addition to these initial tests, which clearly evidenced that the cells in contact with the hydrogel are alive and healthy, the metabolic activity was also evaluated (Figure 3). From the values observed in Figure 3, it can be concluded that all hydrogels were able to host active cell populations. At early stages (96h), only positive charged hydrogels $\left(\right.$ HYD-N ${ }^{+}$) had similar values than TCP control. At later stages, there were no significant differences between samples, showing a good general behavior and similar to the previously described on negatively charged hydrogels. 




Figure 3. Metabolic activity of cell culture at $96 \mathrm{~h}$ and at $168 \mathrm{~h}$ over hydrogels surface. Absolute fluorescence values with mean \pm SD are shown, compared to Treated Control Plastic (TCP) as standard commercial platform for cell growth. TCP value at $168 \mathrm{~h}$ was not represented in a real



In order to employ these hydrogels for cell manipulation, the capability of the formed cell monolayers to be detached and transplanted onto synthetic model substrates was evaluated. Figure 4 shows images of cell transplants (carried out at day 7) at $48 \mathrm{~h}$ after transplant process. In addition, metabolic activity was measured for the transplants equally after $48 \mathrm{~h}$, showing healthy and viable cultures (data not shown). First of all it can be noted that the uncharged hydrogel was not capable of delivering cell monolayers and thus the full transplant could not be successfully achieved. Similarly to the behavior of the previously reported negative hydrogels, all the charged systems were able to spontaneously detach and transplant the cell monolayer (avoiding the use of any chemical or biological agents ) by turning upside down a hydrogel with a cell culture over it and putting in contact with another treated model surface, such as culture treated control plastic (TCP). All of the charged hydrogels maintain the ability of the original 
negative material to be used as a monolayer transplant platform, in spite of the variable ionic moieties. Nevertheless, few differences were observed in the amount of transplanted cells depending on the surface charge. In the case of the hydrogels $\mathrm{HYD}_{-} \mathrm{SO}_{3}^{-}$(negatively charged) and $\mathrm{HYD}-\mathrm{N}^{+}-\mathrm{SO}_{3}{ }^{-}$, monolayers similar to the previously described were obtained ${ }^{4}$. It is remarkable the performance of the hydrogel $H Y D-N^{+}$(positively charged), which produced a large detached monolayer with a dense network of interconnected tissue. Finally, an intermediate situation was found for zwitterionic $\mathrm{HYD}-\mathrm{PO}_{3}^{-}-\mathrm{N}^{+}$, with a better behavior than $\mathrm{HYD}-\mathrm{SO}_{3}^{-}$but lower levels than $\mathrm{HYD}-\mathrm{N}^{+}$.

Thus, it appears that -compared to the uncharged PVP hydrogel- the introduction of charges (negative, zwitterionic and, in particular, positive) over the hydrogels surface caused a general improvement of cell adhesion and proliferation at early stages over the hydrogels. While uncharged PVP confers an intrinsic antifouling behavior, the unspecific introduction of charges may facilitate an unspecific interaction with serum proteins allowing a better cell adhesion ${ }^{23}$. This is somehow surprising for the zwitterionic units (or the pseudozwitterionic, that is the stoichiometric combination of positive and negative) since they are well known non-fouling moieties. Examples may be found in the literature where, to confer surface adhesiveness to zwitterionic polymers, adhesive biomimetic groups are required. ${ }^{24}$ 




Figure 4. Bright field images of cell transplants (Day 7) at $48 \mathrm{~h}$ after transplant process. Extended cell monolayers or big cell patches were observed. (Scale bar: $200 \mu \mathrm{m})$

\section{Bacterial Adhesion tests}

In the context of bacterial colonization, the structures studied in this work are expected to elicit different responses. PVP itself is a neutral and water-soluble polymer that has been grafted to different membranes and materials to confer them antifouling properties. ${ }^{25-30}$ Regarding the introduction of charges, negatively charged interfaces, i.e. similar to the bacterial cell wall, are expected to reduce the affinity of bacteria for the hydrogel surface. Positive quaternary ammonium salts are considered to be efficient antimicrobials, so that they would enable the possibility to reduce the amount of bacteria in the media able to colonize the hydrogel. Finally, based on previous examples reported in the literature we explored electro neutral surfaces produced by the use of two different zwitterionic monomers and finally by incorporating stoichiometric amounts of positive and negatively charged monomers. Zwitterionic monomers have shown remarkable antifouling behavior and their eventual antimicrobial properties are 
currently under investigation. In this study, we considered two different aspects in the use of hydrogels loaded with zwitterionic moieties. On the one hand, the amount of zwitterionic units within the hydrogel structure required to maintain appropriate antifouling properties. On the other hand, we evaluated the role of the position of the positive and negative charge within the monomer unit.

In addition, low modulus of all hydrogels is another favorable parameter to reduce bacterial contamination, as described in the literature. For instance, Cuégan and coworkers ${ }^{22}$ concluded that an increase in the stiffness leads to an enhancement of the number of bacteria on the surface. Equally, Kolewe et al. ${ }^{21}$ evidenced that bacterial attachment onto intermediate to stiff hydrogels is significantly greater than onto soft hydrogels. In particular, Kolewe et al. classify the hydrogels into three different categories depending on the Young modulus. As a result, soft hydrogels are considered those with Young modulus below 1350kPa. Intermediate hydrogels exhibit Young modulus comprised between 1350 and $1500 \mathrm{kPa}$ and finally stiff hydrogels are those with higher modulus. In their study, they evidenced a clear decrease of the bacterial adhesion in soft hydrogels. In spite of the variations on the modulus (see Table 1), the hydrogels obtained can be classified as soft hydrogels independently of the functional monomer incorporated, therefore are in the optimal range to reduce bacterial contamination.

The first series of experiments were conducted by incubating the surfaces with a bacterial suspension at an optical density of 1 during $1 \mathrm{~h}$. As expected, neutral (HYD) and positive (HYD$N^{+}$) charged hydrogels exhibited a large difference in the amount of bacteria immobilized. Based on the antifouling character of the PVP employed to construct the hydrogels, neutral surfaces showed a very low density of immobilized cells of approximately 0.03 cells $/ \mu \mathrm{m}^{2}$. On contrary, positively charged surfaces $H Y D-N^{+}$clearly evidenced a large bacterial surface coverage $\left(0.79\right.$ cells $\left./ \mu \mathrm{m}^{2}\right)$ (Figure 5). This behavior is not surprising taking into account the chemical composition of the bacterial surface. It is already well known that both gram positive and gram negative bacterial cells present a net negative surface charge mostly due to the presence on their cell wall of teichoics acids in Gram positive bacteria, and phospholipids and 
lipopolysaccharides in Gram negative ${ }^{31,32}$. As a result, they are able to interact by electrostatic interactions with positively charged surfaces.

Interestingly, short-term adhesion tests evidenced also a large amount of bacteria immobilized onto negatively charged surfaces $\mathrm{HYD}-\mathrm{SO}_{3}{ }^{-}$. Most probably the salts in the PBS media screen at least initially the repulsion forces between the surface and the bacterial cell wall. It has to be pointed out that $\mathrm{C} 2 \mathrm{C} 12$ mouse muscle myoblastic cells also adhered and proliferated on these negative hydrogels ${ }^{13}$.

In addition to positively and negatively charged surfaces, we have evaluated the synergistic effect of zwitterionic monomers known as antifouling and even antibacterial in combination with the antifouling PVP polymer chains. ${ }^{33,}{ }^{34} \mathrm{We}$ focused on the use of two different kind of zwitterions that either have the positive charge exposed to the external part of the monomer unit,




depicted in Figure 5, in both cases the bacterial attachment remains significantly low (and similar to the neutral hydrogel), in comparison with the previously analyzed positively and negatively charged hydrogels. However it is interesting to note that the hydrogel fabricated using $M-\mathrm{PO}_{3}^{-} \mathrm{N}^{+}$exhibit a slightly larger amount of bacteria $\left(40 \times 10^{5}\right.$ cells/ $\left.\square \mathrm{m}^{2}\right)$ that the analogue $\mathrm{M}-\mathrm{N}^{+}-\mathrm{SO}_{3}{ }^{-}$with the positive charge protected inside the monomer $\left(30 \mathrm{x} 10^{5} \mathrm{cells} / \mu \mathrm{m}^{2}\right)$.

(a)

(b) 



Figure 5. (a) Average bacterial density $\left(\right.$ number/ $\left./ \mathrm{cm}^{2}\right)$ and (b) bacterial surface coverage (\%) quantified from the fluorescence images. For each sample, the columns (each color indicates a particular hydrogel composition) depict the bacterial concentration for $1 \mathrm{~h}$ (left column), $24 \mathrm{~h}$ (center column) and 72 (right column).

(a)

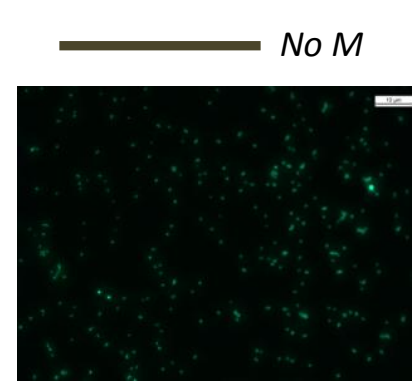

$0.03 \pm 0.02$ cells $/ \mu \mathrm{m}^{2}$ (b)

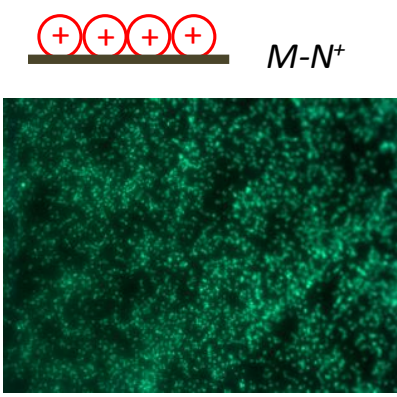

$0.79 \pm 0.07$ cells $/ \mu \mathrm{m}^{2}$ (c)


$0.13 \pm 0.03$ cells $/ \mu \mathrm{m}^{2}$ (d)



$0.03 \pm 0.01$ cells $/ \mu \mathrm{m}^{2}$ (e)

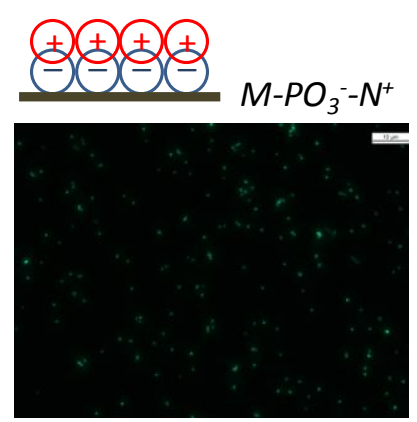

$0.04 \pm 0.01$ cells $/ \mu m^{2}$ 
Figure 6. Representative fluorescence micrographs of the hydrogel surface upon contact with $S$. aureus after $1 \mathrm{~h}$ period. (Bacterial concentration: $\mathrm{OD}=1$ ) (a) Neutral surface $H Y D$ without charged monomer, (b) positively charged hydrogel $H Y D-N^{+}$, (c) negatively charged hydrogel $\mathrm{HYD}-\mathrm{SO}_{3}^{-}$, zwitterionic sulfobetaine $\mathrm{M}^{-} \mathrm{N}^{+}-\mathrm{SO}_{3}^{-}$(d) and phosphorylcholine $\mathrm{M}-\mathrm{PO}_{3}^{-}-\mathrm{N}^{+}$ containing hydrogels (e). As evidenced, the hydrogels bearing either $M-N^{+}$or $M-\mathrm{SO}_{3}{ }^{-}$exhibit an extensive initial bacterial adhesion. Those without any charge (formed by the antifouling PVP) and those incorporating zwitterionic moieties exhibit very low bacterial adhesion at initial stages.

Preliminary tests on the bacterial adhesion at short incubation times were further completed. The surfaces resulting from this initial adhesion were incubated into culture media at longer periods (up to $72 \mathrm{~h}$ ) in order to analyze the effect of the surfaces on bacteria growth and eventually evaluate the biofilm formation. For that purpose, the hydrogels were exposed first to the bacterial dispersion during $1 \mathrm{~h}$. After that the bacterial solution was removed and the hydrogels incubated in culture media during 24 or $72 \mathrm{~h}$.

In Figures $\mathbf{7}$ and $\mathbf{8}$ are depicted representative fluorescence micrographs of $S$. aureus attached on the hydrogels after $1 \mathrm{~h}$ and after incubation for $24 \mathrm{~h}$ and $72 \mathrm{~h}$. Moreover, a comparison of the bacterial density observed at the surface for the different incubation times as a function of the monomer incorporated is shown in Figure 5.

The neutral surfaces $H Y D$ show an increase on the amount of bacteria on the surface as a function of the incubation time in spite of their a priori antifouling properties (Figure 7 and 8). Thus, the bacterial density continuously increases from 0.03 cells $/ \mu \mathrm{m}^{2}$, to 0.29 cells $/ \mu \mathrm{m}^{2}$ after $24 \mathrm{~h}$ and finally to 0.51 cells $/ \mu \mathrm{m}^{2}$ upon $72 \mathrm{~h}$. The positively charged surfaces $H Y D-N^{+}$exhibit a high bacteria density at the different incubation times. For these hydrogels, the surface density remains in values above 0.60 cells $/ \mu \mathrm{m}^{2}$. This result clearly indicates that the bacterial growth in the media exceeds the possible capability of the hydrogel to kill bacteria in contact with the surface. 
A complete substitution of the positively charged monomers by the negatively charged monomers $\left(\mathrm{HYD}-\mathrm{SO}_{3}{ }^{-}\right)$produces significant differences at long incubation times in culture media reducing the cell density from 0.13 cells $/ \mu \mathrm{m}^{2}$ after 1 hour adhesion down to 0.03 cells $/ \mu \mathrm{m}^{2}$ in $72 \mathrm{~h}$ post incubation in culture media. This result might indicate that the initial unexpected high adhesion to the negative surface was mostly due to loosely bound bacteria that are not able to grow on the surface and are released to the media during long incubation times. Interestingly, this behavior has been also observed in our previous work dealing with the cell adhesion on similar surfaces. In those previous experiments, we observed that mouse $\mathrm{C} 2 \mathrm{C} 12$ cells are able to adhere and proliferate on similar surfaces. Moreover, the cell sheet formed were easily detached by mechanical agitation, which indicated a weak interaction of the cells with the underlying substrate in agreement with the decrease at high times in bacterial density observed in this work.

Another interesting case concerns the partial substitution to obtain an average neutral hydrogel $\left(\mathrm{HYD}-\mathrm{N}^{+} / \mathrm{SO}_{3}{ }^{-}\right)$that exhibits very low adhesion. In contrast to the neutral hydrogel $H Y D$, those prepared from stoichiometric amount of positively charged and negatively charged monomers exhibit interesting antifouling properties independently of the incubation time with bacterial densities at the surface below 0.03 cells $/ \mu \mathrm{m}^{2}$. The mechanism for this behavior is still under investigation. However, most probably the presence of distilled water employed as solvent in the photosensitive mixture enables the electrostatic interactions between the opposite charges thus leading to a pseudo zwitterion-like structure. As a consequence, this system resembles, at least to some extent, the situation found when using zwitterionic monomers in the formation of the hydrogels. 




Figure 7. Illustrative images of the evolution of bacterial colonization and biofilm formation on PVP hydrogels with variable surface charge (neutral $H Y D$, positive $H Y D-N^{+}$, negative $H Y D$ $\mathrm{SO}_{3}{ }^{-}$and positive-negative with overall electrical neutrality $\mathrm{HYD}^{-} \mathrm{N}^{+} / \mathrm{SO}_{3}{ }^{-}$) observed upon $72 \mathrm{~h}$. incubation.

Figure 8 shows representative micrographs of the evolution of $S$. aureus attachment to hydrogels having variable density of zwitterionic functional groups as well as the relative position of the charges. Equally, the evolution of the bacterial adhesion to the neutral surfaces HYD is depicted in Figure 8 for comparative purposes. In general, it is possible to observe that the incorporation of zwitterionic groups enhanced the antifouling properties of the hydrogels. 
However, significant differences can be also observed depending on the type and amount of zwitterion employed in particular for long incubation times. As evidenced in Figure 8, hydrogels $\mathrm{HYD}-\mathrm{PO}_{3}^{-}-\mathrm{N}^{+}$show antifouling properties during the first incubation period up to 24h. However, the antifouling effect clearly decreased after $72 \mathrm{~h}$ incubation. As a result the average cell density increased from $0.03-0.04$ cells $/ \mu \mathrm{m}^{2}$ up to 0.1 cells $/ \mu \mathrm{m}^{2}$ when using $12 / 1$ molar ratio $\mathrm{VP} / \mathrm{M}-\mathrm{PO}_{3}-\mathrm{N}^{+}$and 0.4 cells $/ \mu \mathrm{m}^{2}$ in the case of $6 / 1$ molar ratio ${ }^{+}$. More interestingly, the hydrogels $\mathrm{HYD}-\mathrm{N}^{+}-\mathrm{SO}_{3}{ }^{-}$exhibit a different behavior. On one hand, the hydrogels obtained from an initial feed composed of 12/1 molar ratio $\mathrm{VP} / \mathrm{M}-\mathrm{N}^{+}-\mathrm{SO}_{3}{ }^{-}$exhibit a similar behavior when compared to the neutral hydrogels $H Y D$ with an increasing cell coverage starting at 0.02 cells $/ \mu \mathrm{m}^{2}$ after $1 \mathrm{~h}$ up to 0.24 cells $/ \mathrm{mm}^{2}$ upon $72 \mathrm{~h}$ incubation. On the other hand, an increase of the amount in $\mathrm{M}-\mathrm{N}^{+}-\mathrm{SO}_{3}{ }^{-}$using a precursor feed of 6/1 molar ratio $\mathrm{VP} / \mathrm{M}-\mathrm{N}^{+}-\mathrm{SO}_{3}{ }^{-}$significantly improved the antifouling properties. In this case, the cell density remains extremely low with values below 0.03 cells $/ \mu \mathrm{m}^{2}$ after $1 \mathrm{~h}$ adhesion as well as after the long incubation times explored.

In summary, significant differences were observed when using zwitterions depending on the type of molecule employed. This observation has not been evidenced in previous works in which both zwitterionic monomers have been reported to prevent bacterial adhesion. Most probably the different orientation of the positive and negative charges of the monomer side groups is the cause of such differences. On the one hand, $\mathrm{M}_{-} \mathrm{PO}_{3}-\mathrm{N}^{+}$has the positive functional group oriented to the environment and therefore attracts negatively charged bacteria. On the other hand, $\mathrm{HYD}-\mathrm{N}^{+}-\mathrm{SO}_{3}{ }^{-}$has the negative $-\mathrm{SO}_{3}{ }^{-}$groups more exposed (the positive quaternary amine groups are closer to the main chain) and may improve the repulsion against bacterial cells. In conclusion, it appears that the incorporation of additional antifouling moieties clearly improved the PVP properties when exposed to longer incubation times. 




Figure 8. Illustrative images of the evolution of bacterial colonization and biofilm formation on PVP hydrogels prepared using zwitterionic monomers, i.e. 2-methacryloyloxyethyl phosphorylcholine $\quad\left(H Y D-\mathrm{PO}_{3}^{-}-N^{+}\right), \quad$ [2-(methacryloyloxy)ethyl]dimethyl-(3sulfopropyl)ammonium hydroxide $\left(\mathrm{HYD}-\mathrm{N}^{+}-\mathrm{SO}_{3}{ }^{-}\right)$, using two different monomer ratio in the precursor feed: 12/1 (hydrogels with lower zwitterion density) and 6/1 (hydrogels with higher zwitterion density) observed upon $72 \mathrm{~h}$. incubation.

\section{Conclusions}


This manuscript describes the fabrication of hydrogels based on antifouling polyvinylpyrrolidone (PVP) able to form pseudo-DNs. In particular, these hydrogels were evaluated for their potential use in tissue engineering applications focusing on the possibility to permit mammalian cell manipulation while preventing the contamination by bacteria. The synthetic strategy developed allowed us to tune the chemical composition of the hydrogels varying the density as well as the type of functional group incorporated. The hydrogels formed maintain common features that permit the fabrication of highly hydrophilic hydrogels with soft mechanical properties and tunable ionic functionalization. As a result, in this contribution the role of the chemical composition on both the mammalian and bacterial cell adhesion as well as the proliferation on hydrogels with similar swelling capabilities, hydrophilicity and mechanical properties has been elucidated.

Variations on the functional groups incorporated in the hydrogel can be easily achieved by introducing methacrylate monomers carrying the proper charge. Thus, positive, negative, neutral (pseudo-zwitterions) and zwitterionic moieties can be introduced in the hydrogel without significantly alter the mechanical properties of the final material.

Unlike the uncharged hydrogel, all the charged surfaces permitted mammalian cell adhesion and proliferation to confluence, as well as the complete monolayer detachment and transplant. Although the positively charged hydrogel exhibited superior performance in terms of adhesion and proliferation rate, the introduction of any charge favors the adhesion and proliferation processes as compared to the uncharged PVP hydrogel.

In contrast to the improvement observed for cell adhesion and growth for any of the described hydrogels, the nature of the charge has a dramatic influence on the bacterial response. In particular two hydrogels exhibit exceptional both short and long-term antifouling properties based on the combination of a PVP inherent antifouling and either a stoichiometric amount of negatively $\left(\mathrm{M}^{+}-\mathrm{SO}_{3}{ }^{-}\right)$and positively charged $\left(\mathrm{M}^{-} \mathrm{N}^{+}\right)$units or incorporating zwitterionic [2-

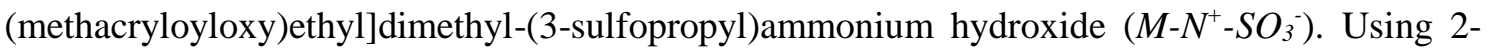





hydrogel did not produce long term antifouling hydrogels.

This study will serve as basis for the development of novel hydrogels with selective properties, i.e. hydrogels able to permit mammalian cell growth and proliferation while preventing bacterial adhesion. Moreover, the formulation of hydrogels with the appropriated functional groups will allow us to store them in aqueous media.

\section{Acknowledgments}

The authors gratefully acknowledge support from the Consejo Superior de Investigaciones Científicas (CSIC). Equally, this work was financially supported by the Ministerio de Economía y Competitividad (MINECO) through MAT2013-47902-C2-1-R, MAT2013-42957-R, MAT2016-78437-R, and BIO2012-34835 and BIO2016-77367 (ALC).

\section{References}

1. Ahmed, E. M., Hydrogel: Preparation, characterization, and applications: A review. Journal of Advanced Research 2015, 6, (2), 105-121.

2. Slaughter, B. V.; Khurshid, S. S.; Fisher, O. Z.; Khademhosseini, A.; Peppas, N. A., Hydrogels in Regenerative Medicine. Advanced Materials 2009, 21, (32-33), 3307-3329.

3. Yin, H.; Akasaki, T.; Lin Sun, T.; Nakajima, T.; Kurokawa, T.; Nonoyama, T.; Taira, T.; Saruwatari, Y.; Ping Gong, J., Double network hydrogels from polyzwitterions: high mechanical strength and excellent anti-biofouling properties. Journal of Materials Chemistry B 2013, $1,(30), 3685-3693$.

4. Aranaz, I.; Martinez-Campos, E.; Nash, M. E.; Tardajos, M. G.; Reinecke, H.; Elvira, C.; Ramos, V.; Luis Lopez-Lacomba, J.; Gallardo, A., Pseudo-double network hydrogels with unique properties as supports for cell manipulation. Journal of Materials Chemistry B 2014, 2, (24), 3839-3848.

5. Zhang, R.; Mjoseng, H. K.; Hoeve, M. A.; Bauer, N. G.; Pells, S.; Besseling, R.; Velugotla, S.; Tourniaire, G.; Kishen, R. E. B.; Tsenkina, Y.; Armit, C.; Duffy, C. R. E.; Helfen, 
M.; Edenhofer, F.; de Sousa, P. A.; Bradley, M., A thermoresponsive and chemically defined hydrogel for long-term culture of human embryonic stem cells. Nature Communications 2013, 4.

6. Reinecke, H.; Gallardo, A., Symmetric Versus Asymmetric "Homologous" Vinylic Cross-Linkers in Two-Components Networks: Formation of Pseudo-Conetworks or PseudoIPNs. Macromolecular Theory and Simulations 2009, 18, (1), 25-29.

7. Gong, J. P.; Katsuyama, Y.; Kurokawa, T.; Osada, Y., Double-network hydrogels with extremely high mechanical strength. Advanced Materials 2003, 15, (14), 1155-1158.

8. Tardajos, M. G.; Aranaz, I.; Pérez, M.; López, D.; Reinecke, H.; Elvira, C.; Gallardo, A., Self-Structuring in Amphiphilic Networks Prepared by Single Conventional Radical Copolymerization of n-Butyl Methacrylate and Vinylpyrrolidone. Macromolecules 2013, 46, (12), 5018-5025.

9. Feng, W.; Brash, J. L.; Zhu, S. P., Non-biofouling materials prepared by atom transfer radical polymerization grafting of 2-methacryloloxyethyl phosphorylcholine: Separate effects of graft density and chain length on protein repulsion. Biomaterials 2006, 27, (6), 847-855.

10. Carr, L.; Cheng, G.; Xue, H.; Jiang, S., Engineering the Polymer Backbone To Strengthen Nonfouling Sulfobetaine Hydrogels. Langmuir 2010, 26, (18), 14793-14798.

11. Shin, Y. S.; Wang, C. M.; Exarhos, G. L., Synthesis of SiC ceramics by the carbothermal reduction of mineralized wood with silica. Advanced Materials 2005, 17, (1), 7377.

12. Yang, B.; Wang, C.; Zhang, Y.; Ye, L.; Qian, Y.; Shu, Y.; Wang, J.; Li, J.; Yao, F., A thermoresponsive poly (N-vinylcaprolactam-co-sulfobetaine methacrylate) zwitterionic hydrogel exhibiting switchable anti-biofouling and cytocompatibility. Polymer Chemistry 2015, 6, (18), 3431-3442.

13. Tardajos, M. G.; Nash, M.; Rochev, Y.; Reinecke, H.; Elvira, C.; Gallardo, A., Homologous Copolymerization Route to Functional and Biocompatible Polyvinylpyrrolidone. Macromolecular Chemistry and Physics 2012, 213, (5), 529-538. 
14. Kwok, D. Y.; Budziak, C. J.; Neumann, A. W., Measurements of static and low-rate dynamic contact angles by means of an automated capillary rise technique. Journal of Colloid and Interface Science 1995, 173, (1), 143-150.

15. Quéré, D.; Di Meglio, J.-M., The meniscus on a fibre. Advances in Colloid and Interface Science 1994, 48, 141-150.

16. Yuan, Y.; Lee, T. R., Contact Angle and Wetting Properties. In Surface Science Techniques, Bracco, G.; Holst, B., Eds. Springer Berlin Heidelberg: 2013; Vol. 51, pp 3-34.

17. Cain, J. B.; Francis, D. W.; Venter, R. D.; Neumann, A. W., Dynamic contact angles on smooth and rough surfaces. Journal of Colloid and Interface Science 1983, 94, (1), 123-130.

18. Yeung, T.; Georges, P. C.; Flanagan, L. A.; Marg, B.; Ortiz, M.; Funaki, M.; Zahir, N.; Ming, W.; Weaver, V.; Janmey, P. A., Effects of substrate stiffness on cell morphology, cytoskeletal structure, and adhesion. Cell motility and the cytoskeleton 2005, 60, (1), 24-34.

19. Engler, A. J.; Richert, L.; Wong, J. Y.; Picart, C.; Discher, D. E., Surface probe measurements of the elasticity of sectioned tissue, thin gels and polyelectrolyte multilayer films: correlations between substrate stiffness and cell adhesion. Surface Science 2004, 570, (1), 142154.

20. Discher, D. E.; Janmey, P.; Wang, Y.-1., Tissue cells feel and respond to the stiffness of their substrate. Science 2005, 310, (5751), 1139-1143.

21. Kolewe, K. W.; Peyton, S. R.; Schiffman, J. D., Fewer Bacteria Adhere to Softer Hydrogels. ACS Applied Materials \& Interfaces 2015, 7, (35), 19562-19569.

22. Guégan, C.; Garderes, J.; Le Pennec, G.; Gaillard, F.; Fay, F.; Linossier, I.; Herry, J. M.; Fontaine, M. N. B.; Réhel, K. V., Alteration of bacterial adhesion induced by the substrate stiffness. Colloids and Surfaces B: Biointerfaces 2014, 114, 193-200.

23. Kikuchi, A.; Okano, T., Nanostructured designs of biomedical materials: applications of cell sheet engineering to functional regenerative tissues and organs. Journal of Controlled Release 2005, 101, (1), 69-84.

24. Zustiak, S. P., Tailoring Hydrogel Adhesiveness to Cells, Proteins, and Bacteria. Gels Handbook: Fundamentals, Properties and Applications (In 3 Volumes) Volume 1: 
Fundamentals of HydrogelsVolume 2: Applications of Hydrogels in Regenerative MedicineVolume 3: Application of Hydrogels in Drug Delivery and Biosensing 2016, 175.

25. Kanagaraj, P.; Nagendran, A.; Rana, D.; Matsuura, T.; Neelakandan, S.; Malarvizhi, K., Effects of Polyvinylpyrrolidone on the Permeation and Fouling-Resistance Properties of Polyetherimide Ultrafiltration Membranes. Industrial \& Engineering Chemistry Research 2015, 54, (17), 4832-4838.

26. Riyasudheen, N.; Paul, M. J.; Sujith, A., Effect of Poly(vinyl pyrrolidone) on Antifouling Properties of Asymmetric Poly(ethylene-co-vinyl alcohol) Membranes. Chemical Engineering \& Technology 2014, 37, (6), 1021-1029.

27. Vatsha, B.; Ngila, J. C.; Moutloali, R. M., Preparation of antifouling polyvinylpyrrolidone (PVP 40K) modified polyethersulfone (PES) ultrafiltration (UF) membrane for water purification. Physics and Chemistry of the Earth 2014, 67-69, 125-131.

28. Feng, R.; Wang, C.; Xu, X.; Yang, F.; Xu, G.; Jiang, T., Highly effective antifouling performance of $\mathrm{N}$-vinyl-2-pyrrolidone modified polypropylene non-woven fabric membranes by ATRP method. Journal of Membrane Science 2011, 369, (1-2), 233-242.

29. Kinnane, C. R.; Such, G. K.; Antequera-Garcia, G.; Yan, Y.; Dodds, S. J.; Liz-Marzan, L. M.; Caruso, F., Low-Fouling Poly(N-vinyl pyrrolidone) Capsules with Engineered Degradable Properties. Biomacromolecules 2009, 10, (10), 2839-2846.

30. Liu, Z. M.; Xu, Z. K.; Wan, L. S.; Wu, J.; Ulbricht, M., Surface modification of polypropylene microfiltration membranes by the immobilization of poly (N-vinyl-2pyrrolidone): a facile plasma approach. Journal of Membrane Science 2005, 249, (1-2), 21-31.

31. Timofeeva, L.; Kleshcheva, N., Antimicrobial polymers: mechanism of action, factors of activity, and applications. Appl Microbiol Biotechnol 2011, 89, (3), 475-492.

32. Siedenbiedel, F.; Tiller, J. C., Antimicrobial Polymers in Solution and on Surfaces: Overview and Functional Principles. Polymers 2012, 4, (1), 46-71.

33. Venault, A.; Zheng, Y.-S.; Chinnathambi, A.; Alharbi, S. A.; Ho, H.-T.; Chang, Y.; Chang, Y., Stimuli-Responsive and Hemocompatible Pseudozwitterionic Interfaces. Langmuir 2015, 31, (9), 2861-2869. 
34. Mi, L.; Jiang, S., Integrated Antimicrobial and Nonfouling Zwitterionic Polymers. Angewandte Chemie International Edition 2014, 53, (7), 1746-1754. 